\title{
Cross-talk Statistics and Impact in Interferometric GNSS-R
}

\author{
Daniel Pascual, Student Member, IEEE, Hyuk Park, Senior Member, IEEE, Raul Onrubia, Student Member, IEEE, \\ Alberto Alonso Arroyo, Student Member, IEEE, Jorge Querol, Student Member, IEEE, and \\ Adriano Camps, Fellow, IEEE
}

\begin{abstract}
This work presents a statistical analysis of the crosstalk phenomenon in interferometric Global Navigation Satellite Systems Reflectometry (iGNSS-R). Cross-talk occurs when the Delay-Doppler Map (DDM) of a tracked satellite overlaps others from undesired satellites. This study is performed for groundbased and airborne receivers, and for a receiver on board the International Space Station (ISS) such as the upcoming GEROSISS experiment. Its impact on ocean altimetry retrievals is analyzed for different antenna arrays. Results show that for elevation angles higher than 60 degrees, cross-talk can be almost permanent from ground, up to $61 \%$ from airborne receivers at $2 \mathrm{~km}$ height, and up to $\sim 10 \%$ at the ISS. Cross-talk can only be mitigated using highly directive antennas with narrow beamwidths. Cross-talk impact using a 7-element hexagonal array still induces large errors on ground, but reduces to centimeter level on airborne receivers, and are negligible from the ISS.
\end{abstract}

Index Terms-Global Navigation Satellite System Reflectometry (GNSS-R), altimetry, cross-talk, Radio Occultation and Scatterometry onboard the International Space Station (GEROS)

\section{INTRODUCTION}

Interferometric GNSS Reflectometry (iGNSS-R) was proposed for mesoscale ocean altimetry to improve the height precision by using the whole bandwidth of the transmitted GNSS signals, and not only the narrow bandwidth of the open civilian signals, as it is done in the conventional method (cGNSS-R) [1], [2]. The iGNSS-R technique presents three major drawbacks as compared to the cGNSS-R one: reduced Signal-to-Noise Ratio (SNR), cross-talk interference coming from undesired GNSS satellites, and larger vulnerability to Radio Frequency Interference (RFI) signals. The impact of the first two problems can be reduced by using highly directive antennas with beam steering in which only the desired satellite is tracked. However, residual interference power from unwanted GNSS satellites may still be received. The RFI mitigation in GNSS-R systems is still under study [3].

Manuscript received MONTH DAY, YEAR; revised MONTH DAY, YEAR. This work was supported by Spanish Research Projects AYA2011-29183C02-01 and ESP2015-70014-C2-1-R from the Spanish Ministry of Economy and Competitiveness, and by the European Union's Seventh Framework Programme for research, technological development and demonstration under grant agreement "European GNSS-R Environmental Monitoring" no FP7607126-E-GEM.

The authors are with the Remote Sensing Laboratory (RSLab), Department of Signal Theory and Communication (TSC), Universitat Politècnica de Catalunya Barcelona-Tech (UPC), 08034 Barcelona, Spain, and also with the Institut d'Estudis Espacials de Catalunya (IEEC)/Universitat Politècnica de Catalunya (UPC), 08034 Barcelona, Spain (e-mail: \{daniel.pascual, park.hyuk, onrubia, alberto.alonso.arroyo, jorge.querol, camps\}@ @tsc.upc.edu).
The most generic observable in GNSS-R is the so-called Delay Doppler Map (DDM), which is the two dimensional cross-correlation in the delay/Doppler plane between the reflected signal and either a clean replica of the transmitted codes in cGNSS-R, or with the direct signal in iGNSS-R. A DDM maps a geographic area into a delay/Doppler space. The mapping depends on the distance and the relative velocity vectors of the vehicles. Its volume, area, center of mass, or symmetry tell about the sea state, wind speed and direction, and other geophysical parameters [2]. The mesoscale altimetry can be retrieved from the position of the maximum of the delay derivative of the DDM [4].

In iGNSS-R, the result of the cross-correlation is not a single DDM, but one DDM for each satellite in view. It may happen that unwanted DDMs fall close to the desired one. This effect is known as cross-talk, and although it has been discussed previously, to knowledge of the authors, has not yet been properly formulated. Cross-talk can introduce critical errors in altimetry, scatterometry, and SAR-like imaging, as well as in defining the correlation window location (i.e. the delay lags in which the cross-correlation is computed) [5], [6].

Figure 1a shows a sketch of a GNSS-R receiver orbiting the Earth with 5 pairs of satellites/specular reflection points in view. The elevation angle $\theta_{e}$ is defined from the receiver's horizontal plane to the GNSS satellite (direct signal elevation angle), unlike in most GNSS-R literature, where the reference point is the specular one. These two angles are almost the same for ground-based and airborne altitudes, but may be different for spaceborne receivers. Five possible iGNSS-R DDMs are drawn in Fig. 1b. If the desired satellite to be tracked is \#1, there would be overlap with the DDMs from satellites \#2 and \#4, while satellites \#3 and \#5 would have no cross-talk impact, although they are also in view.

It is worth to clarify the difference between the crosstalk effect and the result of the cross-correlation between codes that are not totally orthogonal. In cGNSS-R, the later produces an additional Gaussian noise signal for each satellite in view and transmitted code. In the iGNSS-R the number of noise terms is squared. Its impact can be understood as a degradation of the SNR [7]. Results show a reduction smaller than $5 \mathrm{~dB}$ in iGNSS-R with even 20 present satellites when an isotropic antenna is used. In this paper, the powers of the crosscorrelations between different satellites are not considered. However, one can already guess that these terms are much lower than the cross-talk one because the latter has a similar 


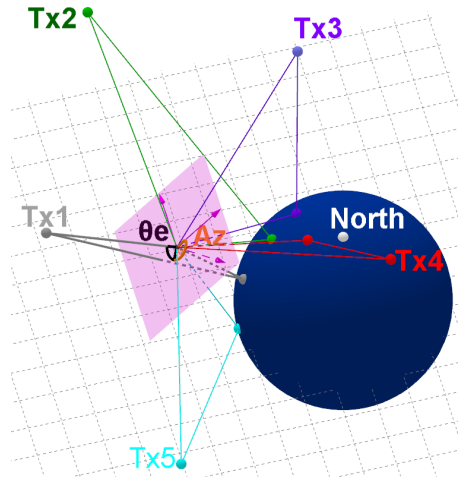

(a)

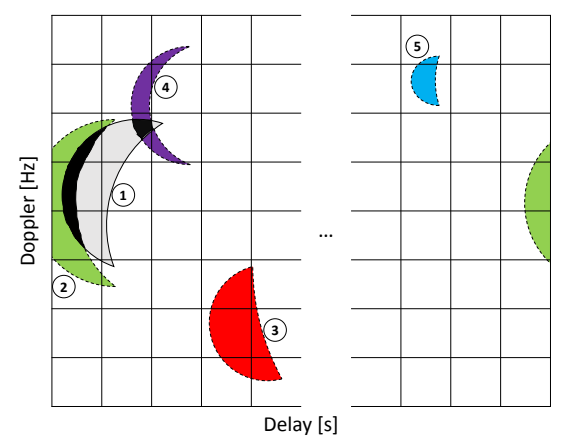

(b)

Fig. 1: Cross-talk phenomenon: (a) Snapshot map view of a GNSS-R scenario with a receiver tracking satellite \#1, and four additional satellites in view (\#2-\#5). (b) DDMs in the delay/Doppler plane. Black areas represent the portions of the desired DDM with cross-talk.

value than the desired cross-correlation.

This work studies the cross-talk probability by simulating 4 consecutive days of GNSS satellites positions sampled each second. The relative position of the interferometric DDMs inside the correlation plane that would be obtained with a receiver is analyzed. The receiver is simulated at different altitudes from ground to airborne, and at the International Space Station (ISS) (orbital height $400 \mathrm{~km}$ ), as it will be the place of a new GNSS-R instrument for the upcoming GNSS rEflectometry, Radio Occultation and Scatterometry onboard International Space Station (GEROS-SS) experiment [8]. The probability of overlapping between DDMs at a certain power is retrieved, as well as the duration statistics of the cross-talk and non cross-talk. Its impact on the specular delay is analyzed by simulating different antenna arrays.

This study is organized as follows: Section II gives a brief summary of the GNSS constellations; Section III gives the expression of the cross-talk occurrence; Section IV analyzes its statistics; Section V studies its impact on GNSS-R retrievals; and finally conclusions are given in Section VI.

\section{GNSS CONSTELLATIONS}

In the coming years, several Global and Regional Navigation Satellite Systems (GNSS and RNSS) constellations will co-exist with more than 140 satellites transmitting simultaneously at different sub-bands inside L-band (1-2 GHz) [9]. The GNSS constellations are: the Global Positioning System (GPS), Galileo, BeiDou-2 (also known as BeiDou Satellite Navigation System or BDS, and formerly known as COMPASS), GLONASS (Russian acronym for Global Navigation Satellite System); in addition to the RNSS: the Indian Regional Navigation Satellite System (IRNSS), and the Japanese Quasi-Zenith Satellite System (QZSS). Most of the GNSS satellites are in Medium Earth Orbit (MEO), but there are also Geostationary Earth Orbit (GEO), GeoSynchronous Orbit (GSO) and inclined GSO (IGSO) satellites at certain locations. The GPS system has also several Satellite Based Augmentation Systems (SBAS) to improve its performance over specific areas. These systems include several stations on ground, as well as GEO or GSO satellites orbiting over certain areas.

In this study, satellite orbits have been propagated with the STK software [10], using their actual ephemeris data. For those satellites that are not launched yet, their nominal ephemeris have been used. It has been assumed that all the satellites transmit in all their available bands, despite of their current version, as it will eventually happen when the constellations get upgraded. A total of $83 \mathrm{MEO}$ satellites (32 GPS, 27 Galileo, and 24 BeiDou-2) have been simulated, as well as 19 satellites with other orbits (11 GPS and 8 BeiDou2). Table I gives the properties of the MEO constellations, and their transmitted signals, and Figure 2 shows their spectra allocation. The Galileo E5A and E5B signals can be processed independently or as a single signal. The powers given in Table I are the minimum received powers on the Earth surface using an isotropic antenna [9]. The relation between these values are used as a reference in the whole paper.

Most of the GNSS signals are actually the aggregate of several codes. In this work, the DDMs are obtained from the whole composite signals, although new techniques to remove specific codes are being investigated [11]. The receiver is simulated to be tuned to each carrier frequency, and with the same bandwidth as the transmitted one. The interfering signals may come from other satellites of the same constellation, and from other constellations if they share the same band. The third row of Table II summarizes the overlapped bands in which cross-talk may happen. If for example the desired signal is a GPS L1 or Galileo E1, the interfering signals may be these two, but also the BeiDou-2 B1 and B1-2. However if the desired signal is a BeiDou-2 B1 or B1-2, it would have interference only with its own constellation. The reason is because the receiver is designed to receive only this specific signal, very little power would be received from the GPS L1 or Galileo E1 [12] .

\section{CROSS-TALK DEFINITION}

In order to study the cross-talk probability, it is important to understand the nature of the DDM correlation. Two close DDMs do not necessary imply that their respective pairs of transmitter/specular points are also close and vice-versa. On the one hand, the Doppler depends on the relative velocity 
TABLE I: GNSS constellations summary. Abbreviations: $i=$ inclination, $e=$ eccentricity, and $a=$ semi-major axis.

\begin{tabular}{|c|c|c|c|c|}
\hline System & Constellation & MEO Parameters & Bands & Power (dBm) \\
\hline \multirow{3}{*}{ GPS } & \multirow{3}{*}{$\begin{array}{l}\text { MEO: Walker } 24 / 6 / 1 \\
+ \text { spare satellites }\end{array}$} & \multirow{3}{*}{$\begin{array}{l}i=55^{\circ} \\
e=0^{\circ} \\
a=26560 \mathrm{~km}\end{array}$} & L1 (1.57542 GHz) & C/A: $-161.5, \mathrm{P}:-164.5, \mathrm{M}:-160.0, \mathrm{C}:-160.0$ \\
\hline & & & L2 $(1.227600 \mathrm{GHz})$ & P: $-164.5, \mathrm{M}:-160.0, \mathrm{C}:-161.5$ \\
\hline & & & L5 $(1.176450 \mathrm{GHz})$ & -157 \\
\hline \multirow{5}{*}{ Galileo } & \multirow{5}{*}{$\begin{array}{l}\text { MEO: Walker } 27 / 3 / 1 \\
+ \text { spare satellites }\end{array}$} & \multirow{5}{*}{$\begin{array}{l}i=56^{\circ} \\
e=0^{\circ} \\
a=29601 \mathrm{~km}\end{array}$} & E1 $(1.57542 \mathrm{GHz})$ & A: -157.0, B: $-157.4, C:-167.4$ \\
\hline & & & E6 $(1.278750 \mathrm{GHz})$ & A: $-155.0, \mathrm{~B}:-158.0, \mathrm{C}:-158.0$ \\
\hline & & & E5A $(1.176450 \mathrm{GHz})$ & -155.0 \\
\hline & & & E5B $(1.207140 \mathrm{GHz})$ & -155.0 \\
\hline & & & E5 $(1.191795 \mathrm{GHz})$ & -152.0 \\
\hline \multirow{4}{*}{ BeiDou-2 } & \multirow{4}{*}{$\begin{array}{l}\text { MEO: Walker 24/3/1 } \\
+ \text { spare satellites } \\
\text { GEO: } 5 \\
\text { IGSO: } 3\end{array}$} & \multirow{4}{*}{$\begin{array}{l}i=55^{\circ} \\
e=0^{\circ} \\
a=27840 \mathrm{~km}\end{array}$} & B1 (1.561098 GHz) & -160.0 \\
\hline & & & B1-2 (1.589740 GHz) & -160.0 \\
\hline & & & B2 $(1.207140 \mathrm{GHz})$ & -160.0 \\
\hline & & & B3 $(1.268520 \mathrm{GHz})$ & -160.0 \\
\hline
\end{tabular}
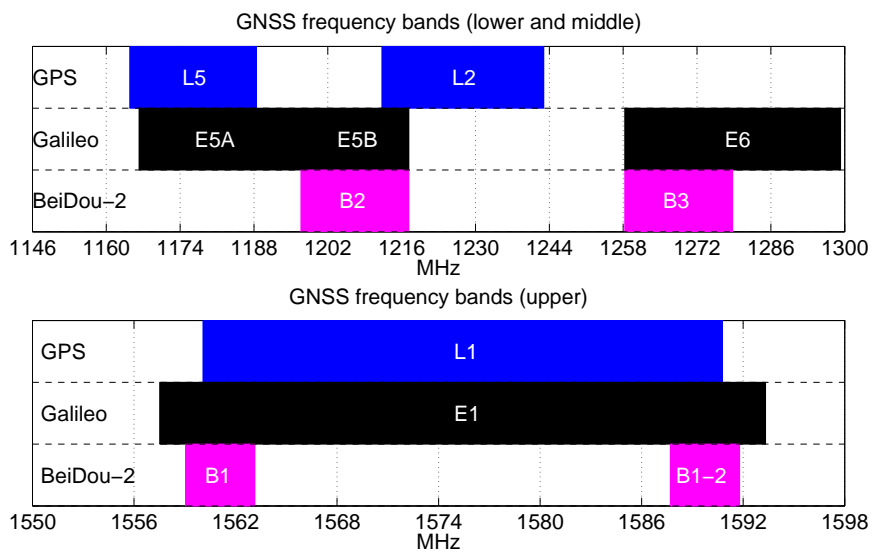

Fig. 2: GNSS frequency bands allocation.

vectors between the transmitter and the receiver. Satellites with different trajectories can have the same Doppler if their radial velocity with respect the receiver is the same. On the other hand, the delay dimension is limited by the minimum between the correlation length $T_{c}$, and the duration of the codes, which can range from $1 \mathrm{~ms}$ to more than one week. However in GNSS-R, the correlation length is often set to $T_{c}=1 \mathrm{~ms}$ to ensure sea state coherence (e.g. [13]). This means that all the interferometric delays multiple of $T_{c}$ fall in the same delay lag (see the DDM \#2 in Fig. 1b).

A DDM can be understood as the two dimensional convolution in the delay and Doppler domains of the Woodward Ambiguity Function (WAF) of the transmitted composite signal with the ocean radar cross-section [2]. The DDM extends the WAF in both domains mainly because of the geometry, but also of the sea state. The size of the WAF in the delay domain is the auto-correlation function (ACF) length $t_{\lambda}$, given in the second row of Table II. The size in the Doppler domain does not depend on the signal but on the inverse of the correlation length $\approx 1 / T_{c}$.

DDMs are simulated using the $\mathrm{P}^{2} \mathrm{EPS}$ software [14], and trimmed within rectangles where their amplitude decays below a threshold value. A satellite has cross-talk if the circumscribed rectangle of its DDM is overlapped with another one or more. It is worth to say that other effects as multipah in the direct and/or the reflected signals [15], or cross-polarization interference, can also result in DDM overlapping. These cases are not considered in this work.

Let $\vec{T}, \vec{R}$ and $\vec{S}$ denote respectively the positions of a GNSS transmitting satellite, a receiver, and their related specular point on the Earth's surface (i.e. the point in which the reflected path is shortest). Their respective velocity vectors are $\overrightarrow{T_{v}}, \overrightarrow{R_{v}}$ and $\overrightarrow{S_{v}}$. The direct signal arrives at the receiver with a delay $\tau_{d}$ and Doppler $f_{d_{d}}$, and the specular reflected signal with $\tau_{r}$ and $f_{d_{r}}$

$$
\begin{aligned}
\tau_{d}= & \frac{|\vec{T}-\vec{R}|}{c}, \\
\tau_{r}= & \frac{|\vec{T}-\vec{S}|}{c}+\frac{|\vec{S}-\vec{R}|}{c}, \\
f_{d_{d}}= & {\left[\left(\overrightarrow{R_{v}}-\overrightarrow{T_{v}}\right) \cdot \frac{(\vec{T}-\vec{R})}{|\vec{T}-\vec{R}|}\right] \frac{f_{c}}{c}, } \\
f_{d_{r}}= & {\left[\left(\overrightarrow{S_{v}}-\overrightarrow{T_{v}}\right) \cdot \frac{(\vec{T}-\vec{S})}{|\vec{T}-\vec{S}|}+\right.} \\
& \left.\left(\overrightarrow{R_{v}}-\overrightarrow{S_{v}}\right) \cdot \frac{(\vec{S}-\vec{R})}{|\vec{S}-\vec{R}|}\right] \frac{f_{c}}{c},
\end{aligned}
$$

where $c$ is the speed of light and $f_{c}$ is the carrier frequency of the transmitted signal. The interferometric Doppler $f_{d}$, and the interferometric delay $\tau$ inside a correlation window of length $T_{c}$, are defined as

$$
\begin{aligned}
f_{d} & =f_{d_{d}}-f_{d_{r}}, \\
\tau & =\left(\tau_{d}-\tau_{r}\right) \bmod T_{c},
\end{aligned}
$$

where mod is the modulus operation. The above equations assume a static scenario and do not take into account the movement of the vehicles during the propagation of the signals. The research done in [16] shows that the errors in 
the true interferometric delay and Doppler frequencies at the ISS are smaller than $0.01 \mathrm{C} / \mathrm{A}$ chips and $0.1 \mathrm{~Hz}$ respectively. At airborne heights, these errors are even much smaller.

For the sake of simplicity, it is assumed that the DDMs are symmetrical in the Doppler axis at $f_{d}$, although the wind speed direction can produce a skewness [17]. The spread in the Doppler domain is then defined as $f_{d} \pm \gamma_{f_{d}}$, which results in a width

$$
F=2 \gamma_{f_{d}}
$$

In the delay domain, the DDM spans from half of the ACF length $t_{\lambda} / 2$ before $\tau$, to $\gamma_{\tau}$ units after $\tau$. The true position of these limits are not necessary before or after because of the circular properties of the delay domain. The geometric delay center $\tau_{c}$, and width $T$ are

$$
\begin{aligned}
\tau_{c} & =\left(\tau+\frac{\gamma_{\tau}-t_{\lambda} / 2}{2}\right) \bmod T_{c}, \\
T & =t_{\lambda} / 2+\gamma_{\tau} .
\end{aligned}
$$

The size of a typical DDM from ground or airborne heights, is similar to the WAF of the transmitted signal: $\gamma_{\tau} \approx t_{\lambda} / 2$, and $\gamma_{f d} \approx 1 \mathrm{kHz}$. From the ISS, the DDMs obtained with $\mathrm{P}^{2} \mathrm{EPS}$ shows $\gamma_{\tau} \approx 6 \mathrm{~L} 1 \mathrm{C} / \mathrm{A}$ chips (a chip is the period of a given code) and $\gamma_{f d} \approx 2 \mathrm{kHz}$ at an amplitude decay at $1 / e$.

Consider two satellites $i$ and $j$. The distance between their interferometric Doppler centers is

$$
\Delta f_{d_{i j}}=\left|f_{d_{i}}-f_{d_{j}}\right| .
$$

In the circular delay space, two distances can be defined between the delay center of their DDMs

$$
\begin{aligned}
& d_{i j}=\left(\tau_{c_{i}}-\tau_{c_{j}}\right) \bmod T_{c}, \\
& d_{j i}=\left(\tau_{c_{j}}-\tau_{c_{i}}\right) \bmod T_{c} .
\end{aligned}
$$

The true delay distance is defined as the minimum of them

$$
\Delta \tau_{i j}=\min \left\{d_{i j}, d_{j i}\right\} .
$$

The two satellites have their DDMs overlapped if $\Delta \tau_{i j}$ and $\Delta f_{d_{i j}}$ are smaller than the defined thresholds

$$
C_{i j}= \begin{cases}1 & \text { if } \Delta \tau_{i j} \leq\left(T_{i}+T_{j}\right) / 2 \text { and } \Delta f_{d_{i j}} \leq\left(F_{i}+F_{j}\right) / 2 \\ 0 & \text { else }\end{cases}
$$

The number of interfering satellites on satellite $i$ is

$$
K_{i}=\sum_{j \neq i} C_{i j}
$$

\section{CROSS-TALK STATISTICS}

This section presents several figures of merit to statistically study the cross-talk behavior. These figures are obtained by averaging the individual statistics of each satellite with all of the same constellation for a specific band. For example, the statistics of the GPS L5 refer to any GPS satellite when the signal of interest is the GPS L5. In this case, the interfering signals may be other GPS L5 signals, but also the Galileo E5A from the Galileo satellites. The statistics are the result of simulating 4 days of satellites positions sampled every second, and are repeated for some receiver altitudes, speeds and locations over the Earth, as well as on the ISS.

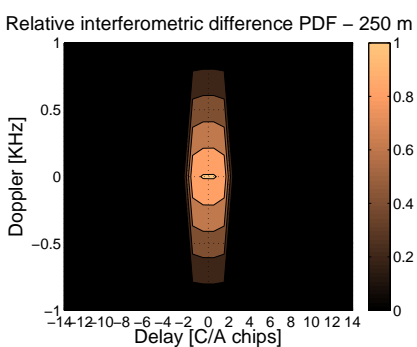

(a)

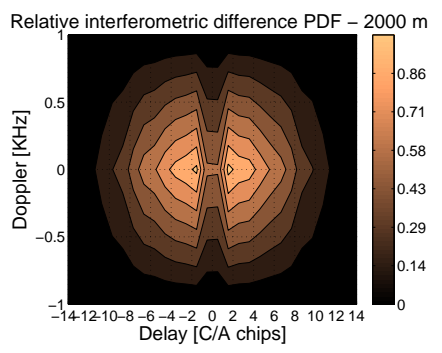

(c)

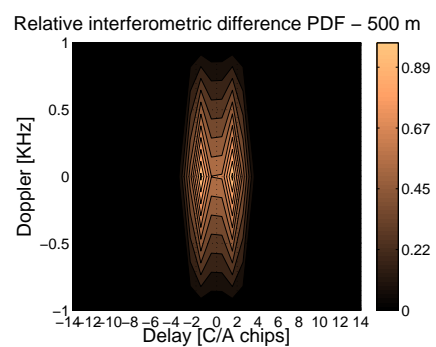

(b)

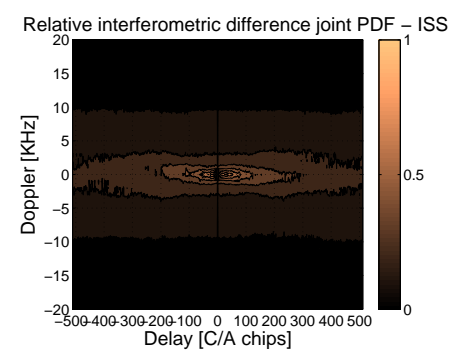

(d)
Fig. 3: Normalized one-to-one joint PDF of the interferometric difference position for the BeiDou-2 B2 signal at different receiver heights (a) $250 \mathrm{~m}$, (b) $500 \mathrm{~m}$, (c) $2 \mathrm{~km}$, and (d) ISS's orbit $(400 \mathrm{~km})$.

\section{A. DDM distribution in the delay/Doppler plane}

The study starts with the one-to-one joint PDF of the interferometric delay and Doppler differences between the tracked satellite and the others in view sharing the same band

$$
P\left(\Delta \tau_{i j}, \Delta f_{d_{i j}}\right) \forall i \neq j .
$$

This PDF gives the distribution in the delay/Doppler plane of the distance between the centers of the desired DDM and the others in view. As an example, Fig. 3 shows the normalized PDF when the tracked satellite is a BeiDou-2, whereas the others may also be Galileo, as it would happen when receiving a BeiDou-2 B2 signal. For low receiver altitudes, the direct and reflected paths are almost equal, which translates in a compensation of the interferometric delay and Doppler. Thus, the interferometric differences are found around the origin of the delay/Doppler plane. As the altitude increases, the direct and reflected paths increase their difference, and the interferometric point move away from the origin. No differences are found for typical vehicle speeds (up to $300 \mathrm{~km} / \mathrm{h}$ ) or locations on Earth. In the ISS case, the delay difference is almost uniform in the delay domain, and the Doppler difference can be up to $10 \mathrm{kHz}$ because of its high speed. Although this PDF does not tell about the existence of cross-talk, it gives the idea that increasing the altitude reduces its probability.

\section{B. Cross-talk probability}

Going into a more detailed analysis, it is interesting to study the probability of cross-talk as function of the elevation angle 


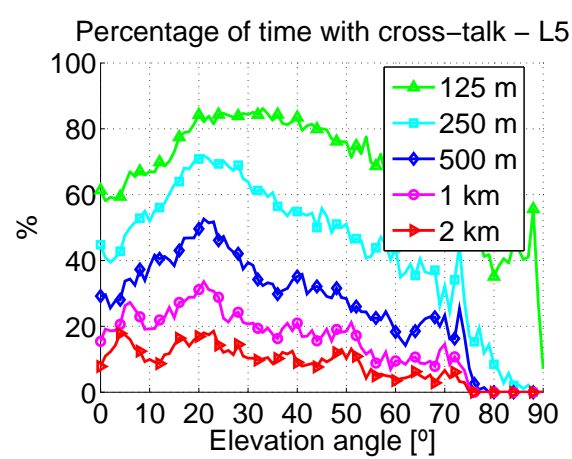

(a)

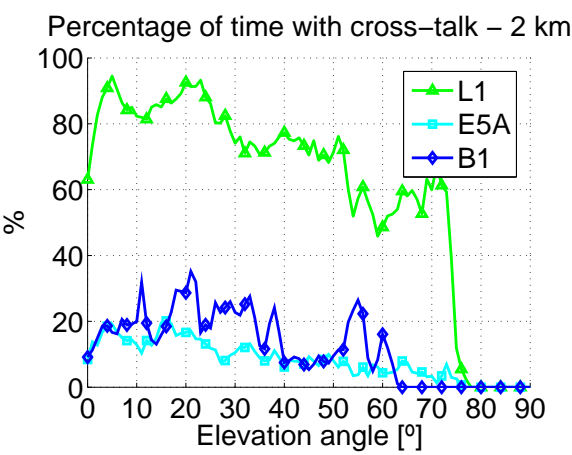

(b)

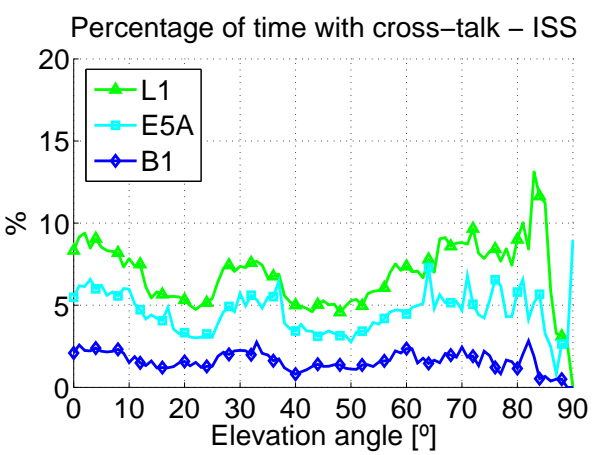

(c)

Fig. 4: Percentage of time with cross-talk as function of the elevation angle of the tracked GNSS satellite. (a) At different receiver altitudes for the GPS L5 band, (b) for the GPS L1, Galileo E5A, and BeiDou-2 B1/B1-2 bands at $2 \mathrm{~km}$ altitude, and (c) at the ISS $(400 \mathrm{~km})$.

of the tracked satellite $i$

$$
P_{c s}\left(\theta_{e}\right)=\frac{1}{C} \sum_{i=1}^{C} P\left\{K_{i}>0 \mid \theta_{e}^{i}=\theta_{e}\right\} .
$$

Figure 4 shows this probability for different receiver altitudes and signals. As a general rule, the probability decreases with the elevation angle. The reason is that most cross-talk happens with satellites with similar elevation angle than the tracked one because their interferometric delay and Doppler differences are similar. Given how the GNSS constellations were designed, it is not common to have many satellites with high elevation angles. On the other hand, and as previously stated, increasing the receiver altitude also reduces cross-talk. From almost constant cross-talk at ground altitudes, to almost zero at airborne scenarios when the elevation angle is high enough $\left(\theta_{e} \approx 65^{\circ}\right)$. The cross-talk probability also depends on the signal band. For example, since the GPS L1 shares the band with the three constellations, it presents more crosstalk probability than the Galileo E5A or BeiDou-2 B1/B1-2, which can only be interfered by two and one constellations respectively. However the Galileo E5A has less cross-talk than the BeiDou-2 B1/B1-2 because the latter has a wider DDM, and thus more chances of overlapping. No differences are observed for different receiver speeds or locations on Earth, except for the fact that the observed elevation angles are limited by the receiver's latitude. The ISS almost constant percentages with $13 \%$ as the worst case.

Most GNSS-R experiments and missions are usually designed to work with satellites with high elevation angles (e.g. [8] up to 35 degrees incidence angles). The first proposed figure of merit is the average cross-talk probability when the elevation angle of the tracked satellite is larger than 60 degrees (but allowing all the angles of the interfering satellites), and averaged over all the satellites of the same constellation

$$
\bar{P}_{c s}^{60}=\frac{1}{C} \sum_{i=1}^{C} \int_{60^{\circ}}^{90^{\circ}} \frac{P\left\{K_{i}>0 \mid \theta_{e}^{i}=\theta_{e}\right\}}{P\left\{\theta_{e}^{i} \geq 60^{\circ}\right\}} d \theta_{e}
$$

where $C$ is the number of satellites of the constellation. The results at $250 \mathrm{~m}, 2 \mathrm{~km}$, and at the ISS are summarized in
Table II. On ground, the percentage of time with cross-talk is almost $100 \%$ for the GPS L1 or Galileo E1 signals, but 19\% for the GPS L2 signal. At $2 \mathrm{~km}$, the largest percentage is also for the Galileo E1 with $61 \%$ of time, whereas for the GPS $\mathrm{L} 2$, it is reduced to $2 \%$. At the ISS, the probabilities are a bit larger than in an airborne case because the DDMs are wider. The Galileo E1 has cross-talk 9.7\% of time and the BeiDou-2 B1 just $1.7 \%$.

\section{Average number of interfering satellites}

The cross-talk can be further analyzed with the number of satellites which are interfering. Let $N_{s a t}^{i}\left(\theta_{e}, K\right)$ be a family of PDFs (on the variable $K$, given in Eq. (15)) for each elevation angle $\theta_{e}$ of the tracked satellite $i$. Figure 5 plots its mode, and $90 \%$ confidence interval for the Galileo E1 and GPS L1 signals at several receiver altitudes. When the altitude is very low, almost all the satellites in view $(\sim 25)$ produce cross-talk, but they are reduced to almost zero when the altitude is over $2 \mathrm{~km}$, and the elevation angle is larger than 60 degrees. At the ISS the cross-talk is mainly produced by a single interfering satellite.

The second figure of merit is the average number of satellites when the elevation angle is larger than 60 degrees, and averaged over all the satellites of the same constellation

$$
\bar{N}_{\text {sat }}^{60}=\frac{1}{C} \sum_{i=1}^{C} \sum_{k=1}^{C+O-1} \int_{60^{\circ}}^{90^{\circ}} \frac{P\left\{K_{i}=k \mid \theta_{e}^{i}=\theta_{e}\right\} \cdot k}{P\left\{\theta_{e}^{i} \geq 60^{\circ}\right\}} d \theta_{e},
$$

where $O$ is the number of satellites of other constellations that may interfere. Results are shown in Table II. At $250 \mathrm{~m}$, the signals GPS L1 and Galileo E1 have an average of 6 interfering satellites, whereas the GPS L2 signal just 0.4 . At $2 \mathrm{~km}$, the GPS L1 and Galileo E1 signals have 1 and 1.4 receptively, while the other signals have less than 0.5 . Finally at the ISS, the maximum average interfering satellites is 0.2 .

\section{Cross-talk duration statistics}

Another interesting parameter is how the cross-talk is distributed in time. Let $\bar{D}_{\tau_{c s}}^{60}(\tau), \bar{D}_{\tau_{n c s}}^{60}(\tau)$ and $\bar{D}_{\tau_{a}}^{60}(\tau)$, be the 


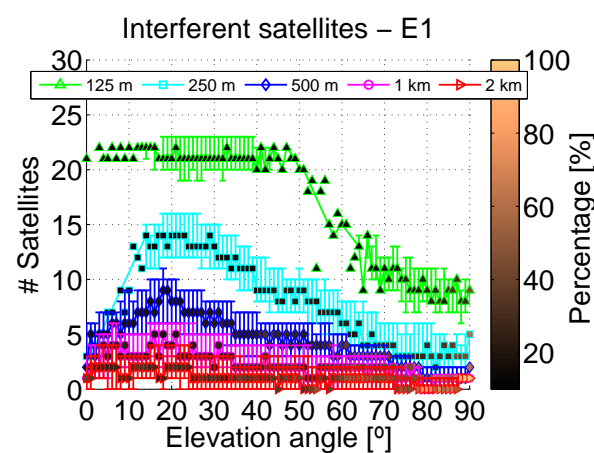

(a)

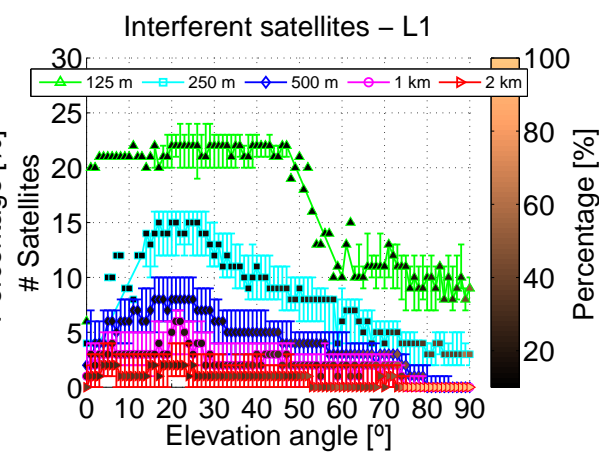

(b)

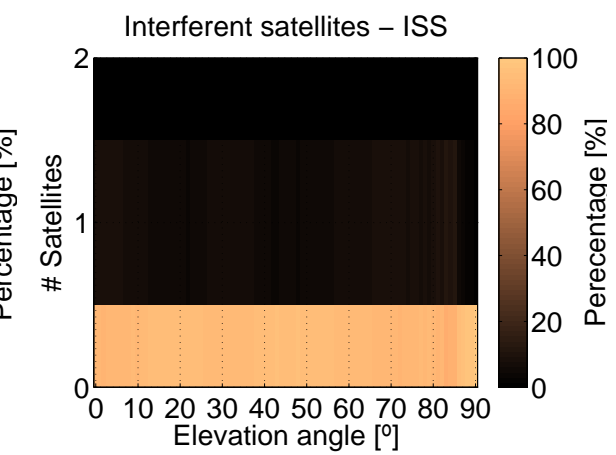

(c)

Fig. 5: Number of interfering satellites (symbols are the mode, and error bars are the $90 \%$ confidence interval) as function of the elevation angle of the tracked GNSS satellite for different receiver altitudes (a) Galileo E1 and (b) GPS L2, and (c) histogram for the GPS L1 at ISS.

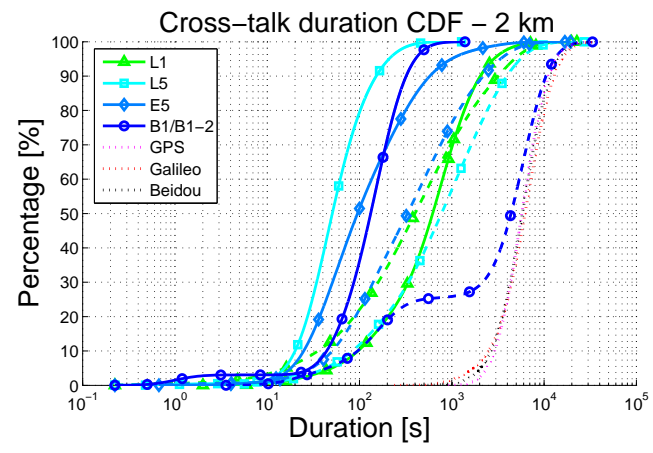

(a)

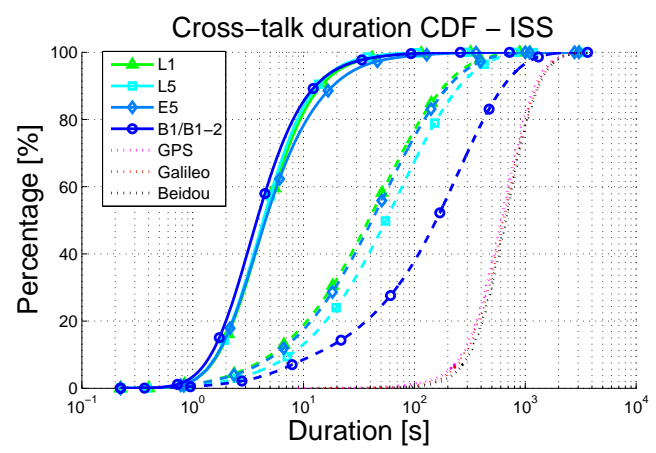

(b)

Fig. 6: Duration CDFs of the cross-talk (solid line with marks), cross-talk free (dashed line), and total access (dotted line) when tracking a GNSS satellite with $\theta_{e}>60^{\circ}$ for the GPS L1 and L5, Galileo E5 and BeiDou-2 B1/B1-2 signals (a) at $2 \mathrm{~km}$, and (b) at the ISS (400 km).

duration CDFs of the cross-talk, cross-talk free, and total access when the elevation angle of the tracked satellite is larger than 60 degrees. The total access duration is defined as the length of time in which the receiver sees the transmitter, and depends on the constellation and not on the individual signals. The CDFs for some bands at $2 \mathrm{~km}$ and on the ISS are plotted in Fig. 6. At these altitudes, in general the cross-talk free duration bursts are longer than the cross-talk ones are, except the GPS L1 at $2 \mathrm{~km}$ which both have a similar behavior. At the ISS, all the cross-talk free bursts are almost the same.

The third and fourth proposed figures of merit are the 90th percentiles $\tau_{c s}$ and $\tau_{n c s}$ of those CDFs

$$
\begin{aligned}
\bar{D}_{\tau_{c s}}^{60}\left(\tau_{c s}\right) & =90 \% \\
\bar{D}_{\tau_{n c s}}^{60}\left(\tau_{n c s}\right) & =90 \%
\end{aligned}
$$

Results are summarized in Table II. For ground-based and airborne receivers, the cross-talk burst duration can be very large, specially for the Galileo E1 signal with 12702 seconds and 2891 seconds respectively, while the largest cross-talk free burst is 1571 seconds for the GPS L2 signal. At the ISS, the cross-talk bursts are reduced to less than 20 seconds, and the cross-talk free are larger than 200 seconds for all the signals. These parameters are useful to define the optimum incoherent averaging duration of the waveforms.

\section{E. Interfering power}

In order to mitigate the cross-talk impact, antenna arrays with beamsteering capabilities and good relative sidelobe levels are needed. Let $G^{i}\left(\theta_{e}, \rho\right)$ be a family of CDFs (given by $\rho$ ) of the Signal-to-Interference Ratio (SIR) between the power of the direct signal of the desired satellite $i$ over all the interfering $j$ after the receiver's up-looking antenna. The SIR for a given realization of a desired satellite is

$$
\rho=\frac{P^{i} \cdot D\left(\theta_{e}^{i}, \phi_{a}^{i}, \theta_{e}^{i}, \phi_{a}^{i}\right)}{\sum_{j \neq i} \rho_{i j}} \cdot\left(\frac{\lambda}{4 \pi\left|\overrightarrow{T^{i}}-\overrightarrow{R^{i}}\right|}\right)^{2},
$$

where

$$
\rho_{i j}= \begin{cases}P^{j} \cdot D\left(\theta_{e}^{i}, \phi_{a}^{i}, \theta_{e}^{j}, \phi_{a}^{j}\right) \cdot\left(\frac{\lambda}{4 \pi\left|\overrightarrow{T^{j}}-\overrightarrow{R^{j}}\right|}\right)^{2} & C_{i j}=1 \\ 0 & \text { else }\end{cases}
$$

where $P$ is the transmitted power, $\phi_{a}$ is the azimuth angle, $D\left(\theta_{e}^{i}, \phi_{a}^{i}, \theta_{e}^{j}, \phi^{j}\right)$ is the receiver's antenna directivity at $\left[\theta_{e}^{j}, \phi_{a}^{j}\right]$ 


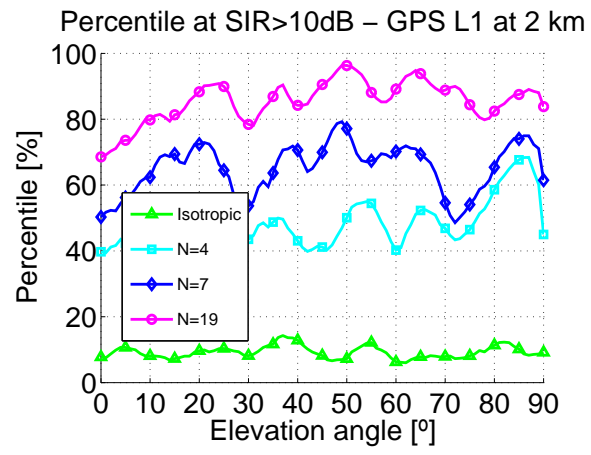

(a)

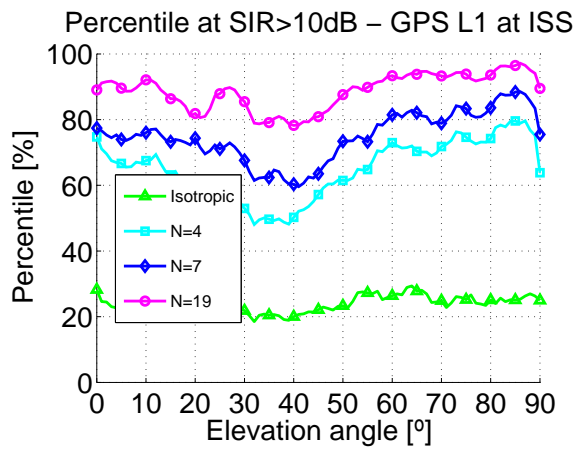

(b)

Fig. 7: Percentile at which SIR $>10 \mathrm{~dB}$ for different antenna arrays for the GPS L1 signal (a) at $2 \mathrm{~km}$, and (b) at the ISS $(400 \mathrm{~km})$.

when pointing at $\left[\theta_{e}^{i}, \phi_{a}^{i}\right]$, and $\lambda$ is the signal wavelength. The performance of different antennas is evaluated by computing the averaged percentile at which the SIR is larger than $10 \mathrm{~dB}$ over all the satellites of the same constellation

$$
\bar{T}\left(\theta_{e}\right)=\frac{1}{C} \sum_{i=1}^{C} G^{i}\left(\theta_{e}, \rho=10 \mathrm{~dB}\right) .
$$

The $10 \mathrm{~dB}$ represents having one or more interfering DDMs whose total power is $1 / 10$ the desired one. This value is an arbitrary one chosen by inspection from the results shown in Figure 7. The degradation of the SNR due to the crosscorrelation between different codes is much lower than this value, and even than the thermal noise [7], and is even more reduced when including the antenna patterns. Figure 7 plots the results for an isotropic antenna, a 4 elements rectangular array, and a 7 and 19 elements hexagonal arrays, all with an element spacing of $0.93 \lambda$. The directivity and beamwidth of the elements is $7.88 \mathrm{~dB}$ and $72^{\circ}$ respectively, similar to the ones designed for the PARIS-IoD mission. The isotropic antenna gives a constant poor performance because it does not mitigate any interfering power, while the antenna arrays improve the SIR by up to 10 at ground, or up to 3 at the ISS. The non-linearity of the array plots, comes from the side lobes which are picking interfering power of satellites with low elevation angles. Similar results are obtained with a spacing of $0.575 \lambda$ to avoid grating lobes in the hexagonal arrays (not shown in the Figures).
The next figure of merit is the average of the percentile over an elevation angle higher than $60^{\circ}$

$$
\bar{T}^{60}=\frac{1}{C} \sum_{i=1}^{C} \int_{60^{\circ}}^{90^{\circ}} G^{i}\left(\theta_{e}, \rho=10 \mathrm{~dB}\right) \frac{P\left\{\theta_{e}^{i}=\theta_{e}\right\}}{P\left\{\theta_{e}^{i} \geq 60^{\circ}\right\}} d \theta_{e} .
$$

Results for the 7 element array are summarized in Table II. At ground, the percentile for the Galileo E1 is just a $6.2 \%$, while the best one is $94.2 \%$ for the BeiDou-2 B1 or B1-2 signals. For an airborne receiver, the former improves to a $58.2 \%$, and to $66.2 \%$ at the ISS

\section{CROSS-TALK IMPACT}

The cross-talk impact on coherent waveforms (i.e. nonaveraged) is evaluated at the point of maximum derivative, as it is a bias of the true altimetry height [4]. The specular point is searched within a narrow window of $+/-100$ meters with respect the theoretical one, as is the largest error between the WGS84, and the geoid over the ocean. Similar to the last section, this study is done statistically and repeated at the same scenarios, and for the different antenna arrays. It is assumed that the desired satellite is ideally pointed by the antennas, and that its DDM is perfectly tracked in the correlation plane.

Figure 8 shows the impact of cross-talk on GPS L1 waveforms, and how the different antenna arrays reduce the interfering waveforms. An isotropic or a single patch antenna would produce indistinguishable corrupted waveforms from which any parameter would be difficult to retrieve. The corrupted waveform at 250 meters, reassembles to a real corrupted one obtained at the TIGRIS experiment [15] at a similar height.

Let $E^{i}\left(\theta_{e}, \epsilon\right)$ and $R^{i}\left(\theta_{e}, \epsilon\right)$ be the PDFs and CDFs of the error $\epsilon$ between the correct specular point and the estimated one of the tracked satellite $i$

$$
\epsilon=|\hat{\tau}-\tau| \text {. }
$$

The average errors are

$$
\bar{\epsilon}^{i}\left(\theta_{e}\right)=\int \epsilon E^{i}\left(\theta_{e}, \epsilon\right) d \epsilon,
$$

and the percentiles at which the error is smaller than $10 \mathrm{~cm}$ are

$$
S^{i}\left(\theta_{e}\right)=1-R^{i}\left(\theta_{e}, \epsilon=10 \mathrm{~cm}\right) .
$$

The last figures or merit are again obtained from the average over an elevation angle larger than 60 degrees

$$
\begin{aligned}
\bar{\epsilon}^{60} & =\frac{1}{C} \sum_{i=1}^{C} \int_{60^{\circ}}^{90^{\circ}} \bar{\epsilon}^{i}\left(\theta_{e}\right) \frac{P\left\{\theta_{e}^{i}=\theta_{e}\right\}}{P\left\{\theta_{e}^{i} \geq 60^{\circ}\right\}} d \theta_{e}, \\
\bar{S}^{60} & =\frac{1}{C} \sum_{i=1}^{C} \int_{60^{\circ}}^{90^{\circ}} S^{i}\left(\theta_{e}\right) \frac{P\left\{\theta_{e}^{i}=\theta_{e}\right\}}{P\left\{\theta_{e}^{i} \geq 60^{\circ}\right\}} d \theta_{e} .
\end{aligned}
$$

Results are presented in Table II when using the hexagonal antenna array of 7-element. The average error at 250 meters can range from $99 \mathrm{~cm}$ for the BeiDou-2 B3 signals to almost zero for the Galileo E5/E5A/E5B signals. The GPS L1 and Galileo E1 have an error of 6.4 and $10.7 \mathrm{~cm}$ respectively. At $2 \mathrm{~km}$, they are reduced to 1 and $2 \mathrm{~cm}$ respectively, while for the other signals the cross-talk has almost disappeared, except for 


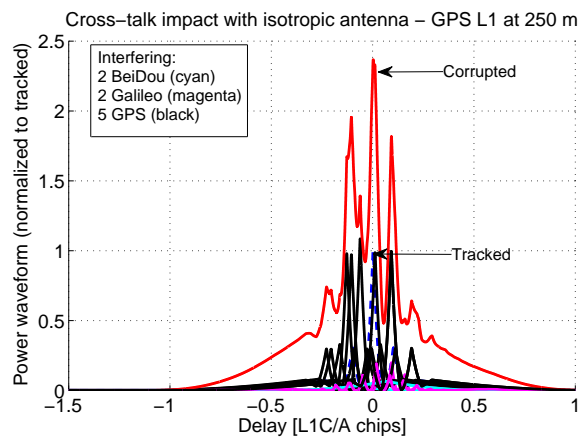

(a)

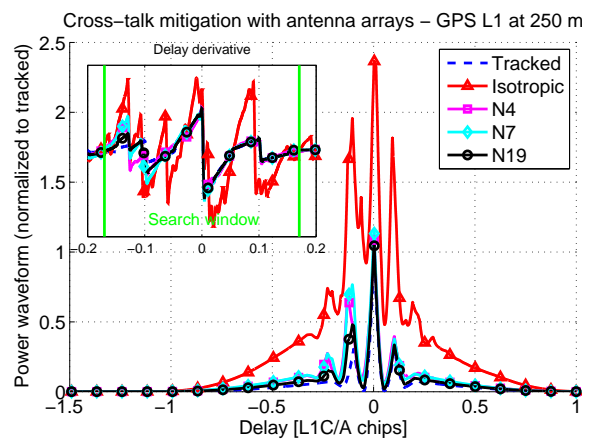

(d)

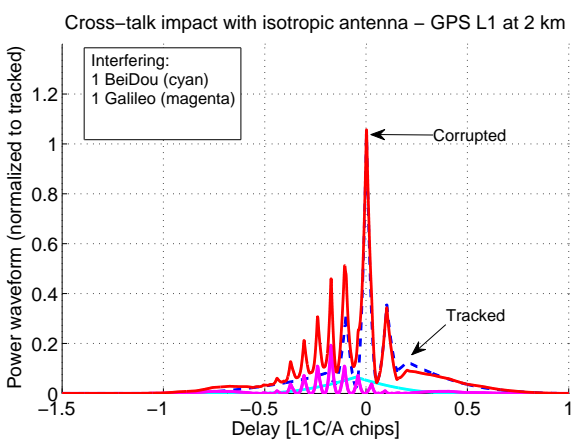

(b)

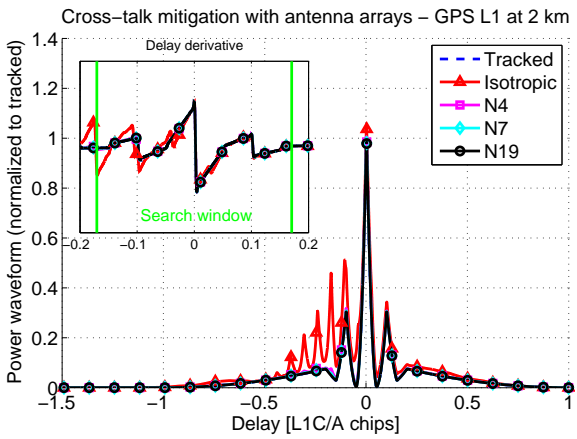

(e)

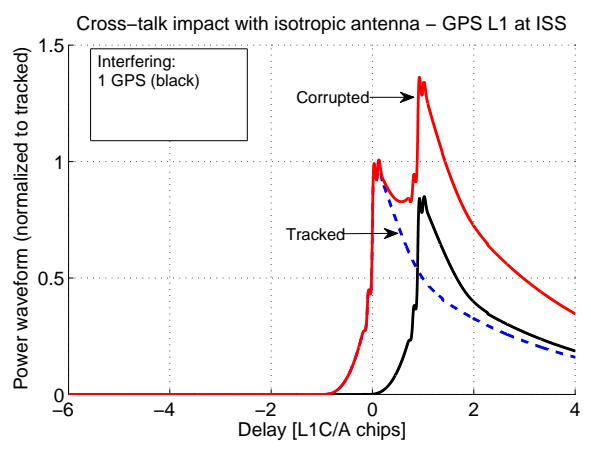

(c)

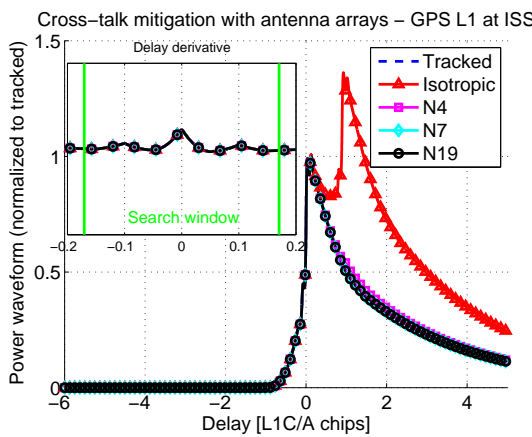

(f)

Fig. 8: Example of cross-talk impact on GPS L1 waveforms. (a)-(c) interfering waveforms and corrupted one obtained with an isotropic antenna at $250 \mathrm{~m}, 2 \mathrm{~km}$, and at the ISS (400 km), and (d)-(f) cross-talk mitigation by using symmetrical antenna arrays of 4,7 and 19 elements.

the BeiDou-2 B2 and B3 signals which still has large errors. At the ISS, all errors are below $0.05 \mathrm{~cm}$.

The percentiles that ensure an error smaller than $10 \mathrm{~cm}$, are found between $13.4 \%$ and $93.3 \%$ at ground for the Galileo E5A and GPS L1 signals respectively. At $2 \mathrm{~km}$ they are between $49.5 \%$ and $98.5 \%$ for the BeiDou-2 B3 and BeiDou2 B1 signals respectively. Although some percentiles may seem to have no improvement with increasing the height, they actually refer to the percentage of time in which there is crosstalk, and is by joining the two factors, that one can observe the enhancement. At the ISS, they are larger than $95 \%$ for all the signals.

The results presented here are very good as it would be expected from the probabilities given in last section. This is because the window used to estimate the specular delay is much smaller than the one defined for the cross-talk overlapping (3 times at ground or airborne, and 21 times at ISS). Searching in the full waveform (e.g. in Fig. 8), produces artificially larger errors of hundreds meters.

\section{CONCLUSIONS}

Cross-talk can be a severe problem in iGNSS-R when all the GNSS constellations will be fully deployed. In order to mitigate it, three requisites should be considered. First, use antenna arrays with beamforming capabilities tracking the target satellite and its reflected point on the surface. Second, known the position of the desired DDM in the delay domain within a window of $+/-100 \mathrm{~m}$. Third, track satellites with elevation angles larger than 60 degrees.

A 7 element hexagonal array is enough to ensure errors below $2 \mathrm{~cm}$ at $2 \mathrm{~km}$ height when tracking satellites with elevation angle larger than 60 degrees. At ground, this antenna still can produce $10 \mathrm{~cm}$ errors, and a larger one, such as a 19 element hexagonal array is recommended.

One could expect that the cross-talk impact from the ISS should be worse, because the statistics shown in Section give a cross-talk probability up to $10 \approx$. The reason of this difference is because the overlapping probability was computed within a window of $[-300,1800]$ meters around the specular point, much larger than the window of $[-100,100]$ meters. Figure $3 \mathrm{~d}$ shows an almost empty area of $[-270,270]$ meters, this tells that actually the interfering DDMs are already quite far from the desired one. In other words, the interfering DDMs overlapp the tracked one with just their tails, instead of with their peak. This principle can also be seen in Figure 8c. This analysis reinforces the GEROS-ISS experiment feasibility.

\section{REFERENCES}

[1] M. Martín-Neira, "A Passive Reflectometry and Interferometry System (PARIS): Application to Ocean Altimetry," J. ESA, vol. 17, 1993.

[2] V. U. Zavorotny, S. Gleason, E. Cardellach, and A. Camps, "Tutorial on Remote Sensing Using GNSS Bistatic Radar of Opportunity," IEEE Geoscience and Remote Sensing Magazine, vol. 2, no. 4, pp. 8-45, Dec. 2014. 
TABLE II: Cross-talk statistics and impact summary when tracking a satellite with $\theta_{e}>60^{\circ}$. Abbrevations are $t_{\lambda}$ : composite signal ACF's length, $\bar{P}_{c s}^{60}$ : cross-talk probability, $\bar{N}_{s a t}^{60}$ : average number of interfering satellites, $\tau_{c s}:$ 90th percentile cross-talk duration, $\tau_{n c s}$ : 90th percentile cross-talk free duration, $\bar{T}^{60}$ : percentile at which SIR is larger than $10 \mathrm{~dB}, \bar{\epsilon}^{60}$ : altimetric average error, and $\bar{S}^{60}$ : percentile at which the error is smaller than $10 \mathrm{~cm}$. Impact results on non-averaged waveforms using a 7 element hexagonal array.

\begin{tabular}{|c|c|c|c|c|c|c|c|c|c|c|c|c|c|}
\hline & Band & GPS L5 & Galileo E5A & Galileo E5B & BeiDou-2 B2 & Galileo E5 & GPS L2 & Galileo E6 & BeiDou-2 B3 & GPS L1 & Galileo E1 & BeiDou-2 B1/B1-2 \\
\hline & & $t_{\lambda}[\mathrm{ms}]$ & 0.2 & 0.2 & 0.2 & 1 & 0.2 & 2 & 0.4 & 0.2 & 2 & 2 & 1 \\
\hline & & Interferent & $\begin{array}{l}\text { GPS L5 } \\
\text { Galileo E5A }\end{array}$ & $\begin{array}{l}\text { GPS L5 } \\
\text { Galileo E5A }\end{array}$ & $\begin{array}{l}\text { Galileo E5B } \\
\text { BeiDou-2 B2 }\end{array}$ & $\begin{array}{l}\text { Galileo E5B } \\
\text { BeiDou-2 B2 }\end{array}$ & \begin{tabular}{|l} 
Galileo E5 \\
GPS L5 \\
BeiDou-2 B2
\end{tabular} & GPS L2 & \begin{tabular}{|l} 
Galileo E6 \\
BeiDou-2 B3
\end{tabular} & \begin{tabular}{|l|} 
Galileo E6 \\
BeiDou-2 B3
\end{tabular} & \begin{tabular}{|l} 
GPS L1 \\
Galileo E1 \\
BeiDou-2 B1 \\
BeiDou-2 B1-2 \\
\end{tabular} & $\begin{array}{l}\text { GPS L1 } \\
\text { Galileo E1 } \\
\text { BeiDou-2 B1 } \\
\text { BeiDou-2 B1-2 } \\
\end{array}$ & $\begin{array}{l}\text { BeiDou-2 } \\
\text { B1/B1-2 }\end{array}$ \\
\hline \multirow{7}{*}{ 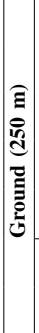 } & \multirow{5}{*}{ 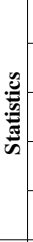 } & $\bar{P}_{c s}^{60}[\%]$ & 30.1 & 29.4 & 73.4 & 86.1 & 80.3 & 99.2 & 51.6 & 50.7 & 100 & 100 & 25.7 \\
\hline & & $\bar{N}_{s a t}^{60}$ & 0.65 & 0.63 & 1.58 & 2.04 & 1.92 & 4.80 & 1.10 & 1.06 & 6.05 & 6.01 & 0.55 \\
\hline & & $\tau_{c s}[s]$ & 282 & 280 & 1198 & 1836 & 1099 & 9470 & 545 & 487 & 10805 & 12780 & 929 \\
\hline & & $\tau_{n c s}[s]$ & 558 & 534 & 435 & 302 & 273 & 272 & 510 & 475 & 98 & 0 & 4161 \\
\hline & & $\bar{T}^{60}[\%]$ & 92.3 & 94.6 & 49.0 & 29.9 & 55.0 & 50.4 & 73.5 & 56.3 & 36.9 & 10.5 & 82.2 \\
\hline & \multirow{2}{*}{ : } & $\bar{\epsilon}^{60}[\mathrm{~cm}]$ & 14.06 & 0.01 & $<0.01$ & 82.12 & $<0.01$ & 3.49 & 0.01 & 127.14 & 19.84 & 2.01 & 0.02 \\
\hline & & $\bar{S}^{60}[\%]$ & 53.8 & 20.3 & 100 & 26.6 & 100 & 99.2 & 100 & 30.3 & 92.0 & 98.3 & 93.7 \\
\hline \multirow{7}{*}{ 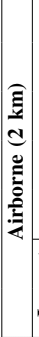 } & \multirow{5}{*}{ 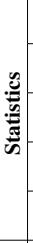 } & $\bar{P}_{c s}^{60}[\%]$ & 3.6 & 3.7 & 20.4 & 24.0 & 22.7 & 27.6 & 12.7 & 13.1 & 44.7 & 61.0 & 2.1 \\
\hline & & $\bar{N}_{s a t}^{60}$ & 0.07 & 0.07 & 0.41 & 0.49 & 0.46 & 0.55 & 0.25 & 0.26 & 1.06 & 1.40 & 0.04 \\
\hline & & $\tau_{c s}[s]$ & 148 & 154 & 800 & 859 & 571 & 2204 & 424 & 432 & 2044 & 2897 & 329 \\
\hline & & $\tau_{\text {ncs }}[s]$ & 3924 & 4250 & 3564 & 3481 & 2193 & 5680 & 3429 & 3355 & 3222 & 2391 & 10397 \\
\hline & & $\bar{T}^{60}[\%]$ & 99.3 & 99.5 & 83.5 & 79.7 & 84.3 & 97.4 & 91.2 & 87.9 & 93.4 & 67.7 & 97.9 \\
\hline & \multirow{2}{*}{ 芯 } & $\bar{\epsilon}^{60}[\mathrm{~cm}]$ & 3.03 & $<0.01$ & $<0.01$ & 78.55 & $<0.01$ & 0.01 & 0.02 & 144.56 & 5.57 & 0.37 & 0.02 \\
\hline & & $\bar{S}^{60}[\%]$ & 73.8 & 40.1 & 100 & 100 & 19.5 & 100 & 100 & 17.1 & 96.3 & 99.4 & 92.3 \\
\hline \multirow{7}{*}{ 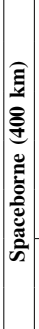 } & \multirow{5}{*}{ : } & $\bar{P}_{c s}^{60}[\%]$ & 5.4 & 5.2 & 6.9 & 7.2 & 9.7 & 2.3 & 6.9 & 7.2 & 8.2 & 9.7 & 1.7 \\
\hline & & $\bar{N}_{s a t}^{60}$ & 0.11 & 0.11 & 0.14 & 0.15 & 0.20 & 0.05 & 0.14 & 0.14 & 0.17 & 0.20 & 0.03 \\
\hline & & $\tau_{c s}[s]$ & 13 & 15 & 22 & 21 & 18 & 13 & 22 & 21 & 14 & 18 & 13 \\
\hline & & $\tau_{n c s}[s]$ & 257 & 278 & 326 & 321 & 205 & 493 & 326 & 321 & 188 & 205 & 638 \\
\hline & & $\bar{T}^{60}[\%]$ & 98.1 & 98.4 & 96.1 & 95.2 & 95.5 & 99.5 & 96.4 & 95.0 & 97.4 & 95.4 & 99.6 \\
\hline & \multirow{2}{*}{. } & $\bar{\epsilon}^{60}[\mathrm{~cm}]$ & 0.26 & 0.01 & 0.02 & 1.42 & 0.01 & 0.04 & 0.02 & 1.42 & 0.09 & 0.07 & 0.11 \\
\hline & & $\bar{S}^{60}[\%]$ & 57.9 & 44.0 & 100 & 97.3 & 100 & 99.9 & 100 & 96.8 & 99.8 & 99.8 & 99.8 \\
\hline
\end{tabular}

[3] J. Querol, G. F. Forte, and A. Camps, "Study of RFI signals in protected GNSS bands generated by common electronic devices: Effects on GNSS-R measurements," in Proceedings of IEEE International Geoscience and Remote Sensing Symposium (IEEE-IGARSS), July1318 2014, pp. 4050-4053.

[4] A. Rius, E. Cardellach, and M. Martín-Neira, "Altimetric Analysis of the Sea-Surface GPS-Reflected Signals," IEEE Transactions on Geoscience and Remote Sensing Letters, vol. 48, no. 4, pp. 2119-2127, 2010.

[5] H. Park, E. Valencia, A. Camps, A. Rius, S. Ribo, and M. MartínNeira, "Delay Tracking in Spaceborne GNSS-R Ocean Altimetry," IEEE Geoscience and Remote Sensing Letters, vol. 10, no. 1, pp. 57-61, 2013.

[6] F. Martin, S. D'Addio, A. Camps, and M. Martín-Neira, "Modeling and Analysis of GNSS-R Waveforms Sample-to-Sample Correlation," IEEE Journal on Selected Topics and Applications, vol. 7, no. 5, pp. $1545-1559,2014$.

[7] Jorge Querol, Alberto Alonso Arroyo, Raul Onrubia, Daniel Pascual, Hyuk Park, and Adriano Camps, "SNR degradation in GNSS-R measurements under the effects of Radio-Frequency Interference," IEEE Journal of Selected Topics in Applied Earth Observations and Remote Sensing.

[8] J. Wickert et al., "GEROS - GNSS REflectometry, Radio Occultation and Scatterometry onboard International Space Station (GEROS-ISS)," in Living planet symposium (ESA), 2013.

[9] Navipedia webpage, ," http://www.navipedia.net/index.php/Main_Page, [last visited on 03/09/15]

[10] Systems Tool Kit (STK) - Analytical Graphics, Inc., ," http://www.agi. com/products/stk/, [last visited on 03/09/15].

[11] W. Li, D. Yank, S. D'Addio, and M. Martín-Neira, "Partial Interferomet- ric Processing of Reflected GNSS Signals for Ocean Altimetry," IEEE Geoscience and Remote Sensing Letters, vol. 9, no. 11, pp. 1509-1513, Sept. 2014.

[12] D. Pascual, H. Park, A. Camps, A. Alonso-Arroyo, and R. Onrubia, "Simulation and Analysis of GNSS-R Composite Waveforms Using GPS and Galileo Signals," IEEE Journal on Selected Topics and Applications, vol. 7, no. 5, pp. 1461-1468, 2014.

[13] G. Foti, C. Gommenginger, P. Jales, M. Unwin, A. Shaw, C. Robertson, and J. Roselló, "Spaceborne GNSS reflectometry for ocean winds: First results from the UK TechDemoSat-1 mission," Geophysical Research Letters, vol. 42, no. 13, pp. 5435-5441, July 2015.

[14] H. Park, A. Camps, D. Pascual, A. Alonso, F. Martin, and H. CarrenoLuengo, "Improvement of the PAU/PARIS End-To-End Performance Simulator (P2EPS) in Preparation for Upcoming GNSS-R Missions," in Proceedings of IEEE International Geoscience and Remote Sensing Symposium, 2013.

[15] F. Martin, A. Camps, F. Fabra, A. Rius, M. Martín-Neira, S. D’Addio, and A. Alonso Arroyo, "Mitigation of Direct Signal Cross-Talk and Study of the Coherent Component in GNSS-R," IEEE Transactions on Geoscience and Remote Sensing Letters, vol. 12, no. 2, pp. 279-283, Feb. 2015

[16] Hyuk Park, Daniel Pascual, Adriano Camps, Francisco Martin, Alberto Alonso-Arroyo, and Hugo Carreno-Luengo, "Analysis of Spaceborne GNSS-R Delay-Doppler Tracking," IEEE Journal of Selected Topics in Applied Earth Observations and Remote Sensing, pp. 1-12, 2014.

[17] E. Valencia, V. Zavoronty, D. M. Akos, and A. Camps, "Using DDM Asymmetry Metrics for Wind Direction Retrieval From GPS OceanScattered Signals in Airborne Experiments," IEEE Transactions on 
Geoscience and Remote Sensing, vol. 52, no. 7, pp. 3924-3936, July 2014.

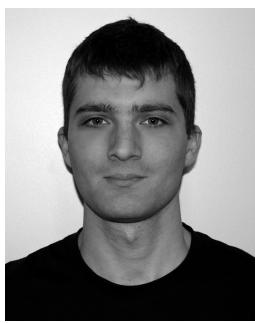

Daniel Pascual (S'11) was born in Barcelona, Spain, in 1985 . He received the $\mathrm{BSc}+5$ degree in telecommunications engineering specialized in communications in 2011, and the MSc+2 degree in Research on Information and Communication Technologies in 2014, both from the Universitat Politècnica de Catalunya (UPC), Barcelona, Spain. In 2011 he joined the Passive Remote Sensing Group from UPC where he is currently working toward the PhD degree in GNSS-Reflectometry focused in ocean altimetry.

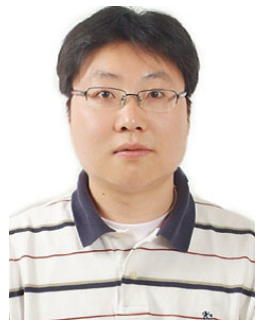

Hyuk Park (S'05-AM'09-M'12) was born in South Korea. He received the B.S. degree in mechanical engineering from the Korea Advanced Institute of Science and Technology (KAIST) in 2001, and received the M.S. and Ph.D. degree in information and mechatronics from the Gwangju Institute of Science and Technology (GIST), Korea, in 2003 and 2009, respectively. His main research interest is in the area of remote sensing, especially passive microwave remote sensing, including system design, modeling and simulation, and image processing. In 2009, he joined the remote sensing group of the Polytechnic University of Catalonia (UPC), Barcelona, as a postdoctoral researcher. He is a grant holder of NRF funded by Korean government in 2011. From 2012, he has been working as a research associate with Grant of Juan de la Cierva funded by Spanish Ministry of Economy and Competitiveness. Currently, he is working with the passive remote sensing group in the UPC for satellite remote sensing for microwave radiometry and GNSS-R.

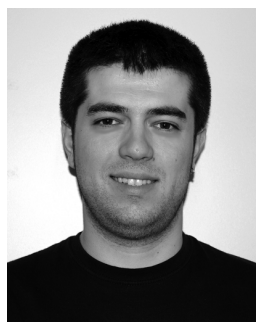

Raul Onrubia (S'10)was born in Barcelona, Spain. $\mathrm{He}$ received the B.Sc. degree $(\mathrm{BSc}+5)$ in telecommunications engineering the M.Sc. degree $(\mathrm{Msc}+2)$ in research on information and communication technologies from the Universitat Politécnica de Catalunya, Barcelona, Spain in 2012 and 2014, respectively. He is currently working toward the $\mathrm{PhD}$. degree in GNSS-Reflectometry with the Passive Remote Sensing Group, Signal Theory and Communications Department, Universitat Politècnica de Catalunya-BarcelonaTech, Barcelona, Spain. His current work is the development of RF hardware, and the study of interferences and mitigation techniques.

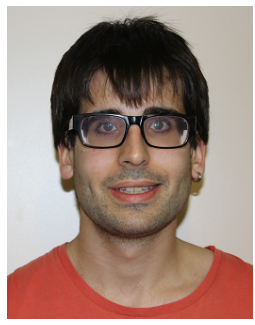

Alberto Alonso Arroyo (S'11) was born in Barcelona, Spain. He received the M.S. degree in telecommunications engineering in $2011(\mathrm{BSc}+5)$ and the M.S. in Research on Information and Communication Technologies in $2012(\mathrm{MSc}+2)$, both from the Universitat Politècnica de CatalunyaBarcelonaTech. He is working toward the Ph.D. degree in GNSS-Reflectometry, with the Passive Remote Sensing Group, Department of Signal Theory and Communications, at the Universitat Politècnica de Catalunya-BarcelonaTech. Currently, he is at National Oceanic and Atmospheric Administration (NOAA) as an invited visiting researcher thanks to a Fulbright grant.

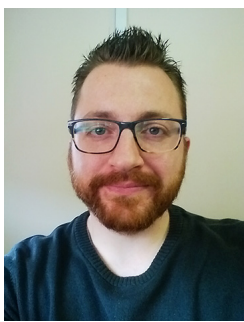

Jorge Querol (S'13) was born in Castelló, Spain, in 1987. He received the M.Sc. degree in electronics engineering, M.Sc. degree in telecommunication engineering and M.Sc. degree in photonics from the UPC-BarcelonaTech in 2011, 2012 and 2013, respectively. Currently, he is a Ph.D. candidate working as graduate research assistant at the Remote Sensing Laboratory (RSLab) at UPCBarcelonaTech, Barcelona, Spain. His research deals with the development of real-time signal processing systems able to detect and mitigate the effects of Radio-Frequency Interference (RFI) signals and jamming in Global Navigation Satellite Systems (GNSS) and Passive Remote Sensing (PRS) applications, particularly, those ones working in L-band such as MicroWave (MW) radiometry and GNSS-Reflectometry (GNSS-R).

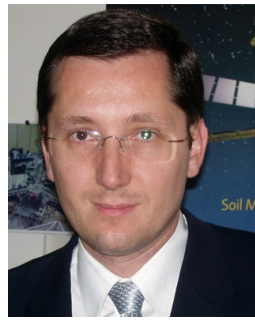

Adriano Camps (S'91-A'97-M'00-SM'03-F'11) was born in Barcelona, Spain, in 1969. He received the degree in Telecommunications Engineering and the Ph.D. degree in telecommunications engineering from the Universitat Politècnica de Catalunya (UPC), Barcelona, Spain, in 1992 and 1996, respectively. From 1991 to 1992 , he was with the ENS des Télécommunications de Bretagne, France, with an Erasmus Fellowship. Since 1993, he has been with the Electromagnetics and Photonics Engineering Group, Department of Signal Theory and Communications, UPC, where he was an Assistant Professor first, an Associate Professor in 1997, and a Full Professor since 2007. In 1999, he was on sabbatical leave at the Microwave Remote Sensing Laboratory, University of Massachusetts, Amherst, MA, USA. Since 1993, he has been deeply involved in the European Space Agency SMOS Earth Explorer Mission, from the instrument and algorithmic points of view, performing field experiments and more recently studying the use of Global Navigation Satellite Systems Reflectometry (GNSSR) techniques to perform the sea state correction needed to retrieve salinity from radiometric observations. His research interests are focused in microwave remote sensing, with special emphasis in microwave radiometry by aperture synthesis techniques, remote sensing using signals of opportunity (GNSSR), and the development of nanosatellites to test innovative remote sensing techniques. Dr. Camps served as Chair of MicroCal (uCal) 2001, Technical Program Committee Co-chair of IEEE International Geosciences and Remote Sensing Symposium (IGARSS) 2007, and Co-chair of GNSS-R 2010. He was an Associate Editor of Radio Science, and he is an Associate Editor of the IEEE Transactions on Geoscience and Remote Sensing and the President-Founder of the IEEE Geoscience and Remote Sensing Chapter in Spain. He was a recipient of the Second National Award of University Studies, in 1993; the INDRA Award of the Spanish Association of Telecommunication Engineers to the Best Ph.D. in Remote Sensing, in 1997; the Extraordinary Ph.D. Award at the UPC, in 1999; the Research Distinction of the Generalitat de Catalunya, for contributions to microwave passive remote sensing, in 2002; the European Young Investigator Award in 2004; and the Institució Catalana de Recerca i Estudis Avançats (ICREA) Academia Award in 2009 and 2014. Moreover, as a member of the Microwave Radiometry Group, UPC, he received the 1st Duran Farell and the Ciutat de Barcelona Awards for Technology Transfer, in 2000 and 2001, respectively, and the "Salvà i Campillo" Award of the Professional Association of Telecommunication Engineers of Catalonia for the most innovative research project for Microwave Imaging Radiometer by Aperture Synthesis/Soil Moisture and Ocean Salinity mission (MIRAS/SMOS)-related activities, in 2004, and the 7th Duran Farell Award for Technological Research, for the work on GNSS-R instrumentation and applications, in 2010. 